\title{
Analysis of cause-of-death mortality and actuarial implications
}

\author{
Hyuk-Sung Kwon ${ }^{1, a}$, Vu Hai Nguyen ${ }^{a}$ \\ ${ }^{a}$ Department of Statistics and Actuarial Science, Soongsil University, Korea
}

\begin{abstract}
Mortality study is an essential component of actuarial risk management for life insurance policies, annuities, and pension plans. Life expectancy has drastically increased over the last several decades; consequently, longevity risk associated with annuity products and pension systems has emerged as a crucial issue. Among the various aspects of mortality study, a consideration of the cause-of-death mortality can provide a more comprehensive understanding of the nature of mortality/longevity risk. In this case study, the cause-of-mortality data in Korea and the US were analyzed along with a multinomial logistic regression model that was constructed to quantify the impact of mortality reduction in a specific cause on actuarial values. The results of analyses imply that mortality improvement due to a specific cause should be carefully monitored and reflected in mortality/longevity risk management. It was also confirmed that multinomial logistic regression model is a useful tool for analyzing cause-of-death mortality for actuarial applications.
\end{abstract}

Keywords: actuarial model, annuity, cause-of-death mortality, life insurance, longevity risk, mortality risk, multinomial logistic regression model

\section{Introduction}

Mortality is an essential consideration in the actuarial risk management of life insurance policies, annuity products, and pension plans as well as fundamental variables in pricing and valuation. Increased life expectancy due to the development of medical and pharmaceutical sciences is a recent life expectancy trend. It implies that life insurers are exposed to longevity risk wherein the expected value of expected annuity or pension benefit is underestimated. In this context, various mortality studies have been performed to understand the nature of human mortality.

The mortality differential measure and its trends exists because there are many aspects numerous factors affecting mortality. These factors can be classified into two categories. One group of factors is associated with risk variables of each individual. Previous mortality studies found that mortality rates are different based on demographic/socioeconomic factors, such as sex, age, marital status, and income level, and health behavioral factors, such as smoking, alcohol intake, dietary habit, and degree of physical activities. Another group of factors comprises external forces that simultaneously affect overall mortality in a certain area. For example, the development of a cure for a specific disease will decrease overall mortality versus a natural disaster or a pandemic in a region that increases mortality temporarily.

Particularly, external forces associated with the remedy of various diseases have significantly increased human mortality in the past several decades. Since a main cause is usually identified and

\footnotetext{
${ }^{1}$ Corresponding author: Department of Statistics and Actuarial Science, Soongsil University, 369 Sangdo-ro, Dongjak-gu, Seoul 06978, Korea. E-mail: hskwon@ssu.ac.kr

Published 30 November 2019 / journal homepage: http://csam.or.kr

(C) 2019 The Korean Statistical Society, and Korean International Statistical Society. All rights reserved.
} 
recorded for each death, the analysis of mortality by the cause of death will provide additional insight for understanding mortality and its trends. Data associated with the main diseases leading to death can also be used to design insurance products covering multiple diseases such as a critical illness insurance policy. Further, an impact of the decrease in mortality due to a specific cause on overall mortality can be quantified using an appropriate mortality model that accommodate various causes of death.

This study closely analyzes mortality by considering the main causes of death. A multinomial logistic regression model was constructed by exploring the historical cause-of-death data in Korea and the US. The model quantified the impact of a change in mortality due to a specific cause on actuarial values of life insurance policies and annuity products; that contribute in quantifying mortality/longevity risks. The model discussed in this study improved the parsimony of the cause-of-death mortality model presented in previous studies by using unabridged mortality data, which also allowed the quantification of the impact of change in mortality level due to a specific cause on actuarial values.

The remaining of this paper is organized as follows. Previous studies regarding cause-of-death mortality are briefly reviewed in Section 2. After the data were introduced and described in Section 3 , the multinomial logistic regression models, which were fitted to the data are discussed in Section 4. Finally, the results of actuarial analyses based on the developed model are shown and their implications are discussed in Section 5. The paper closes with summary and conclusion in Section 6.

\section{Literature review}

Recent studies on human mortality, in a variety of disciplines, have discussed various mortality characteristics such as risk factors on mortality, cause-of-death mortality, mortality improvement, and cohort effect. Especially, actuarial research on mortality focuses on the evaluation of mortality/longevity risk associated with life insurance policies, annuity, and pension plans, reflecting the characteristics of mortality and the trends in experience data. Among various subjects regarding mortality study, the analysis of cause-of-death mortality provides a comprehensive understanding of and additional insight into mortality by allowing an exploration of the impact of the change in the level of mortality due to a specific cause in the overall mortality as well as life expectancy.

The process of collecting cause-of-death data and possible applications for the data were discussed in Israel et al. (1986). Yun and Son (2016) projected future mortality due to various causes, using age-period-cohort model fitted to mortality data in Korea. The authors emphasized that the analysis of cause-of-death mortality is important to estimate future medical costs and establish a preventive policy for a certain cause of death. McNown and Rogers (1992) constructed a multi-exponential model combined with time series model to project the cause-specific mortality of the five main causes of death based on US data. Additionally, past trends in the two leading causes of mortality, heart disease, and cancer were observed in Heron and Anderson (2016). The authors found that the gap in mortality levels between the two causes have changed over time and the changes were different by region and race. Bourmezoued et al. (2018) also studied the effect of change in cause-of-death mortality on population structure. They used cause-of-mortality and fertility data in France and derived the dependency ratio.

Studies on the overall mortality projection based on cause-of-death mortality can be found in Wilmoth (1995) and Richards (2009). Wilmoth (1995) projected overall mortality based on separately estimated mortality rates for various causes using mortality data in Japan. Richards (2009) discussed several issues that arise when overall mortality is projected by summing up cause-specific mortality rates. The author showed that changes in classification of diseases must be appropriately adjusted. 
Arias et al. (2013) derived multiple decrement mortality tables by sex and race after eliminating main causes of mortality based on cause-of-death mortality data in the US. The authors observed that the impact of elimination was greater for cancer and heart disease.

Additionally, there are studies on multiple causes of mortality and correlation among the causes. Manton et al. (1976) analyzed the relationship among multiple causes of death using the lethal defectwear model. Goodman et al. (1982) discussed the usefulness of comprehensive analyses of the multiple causes of death by extracting information on multiple causes of death from death certificates. Moreno-Betancur et al. (2017) suggested a cox-regression model to explain cases where multiple causes of death were observed. Desesquelles et al. (2012) explored mortality data in France and Italy to classify patterns of how multiple causes affect cancer mortality.

Correlations among causes of death have been investigated by several studies. Foreman et al. (2016) suggested a Bayesian hierarchical model to reflect correlations among various causes of death. The authors illustrated the model using Vital Statistics in the US. Copula models were utilized to explain correlations among causes of death. Dimitrova et al. (2013) analyzed mortality data in EnglandWales on the basis of a multivariate copula model. They suggested that the model can be used to perform a scenario test and sensitivity analysis according to the level of cause-of-death mortality. Hong and Yang (2019) introduced the hierarchical Archimedean copula model to project future mortality, considering correlations among causes of death. The authors used cohort mortality data in the US to measure the impact of the change in cancer mortality on overall mortality. Arnold and Sherris (2013, 2015) discussed the Vector Error Correlation Model (VECM) for modeling correlations among the causes of death. They analyzed mortality data in various developed countries, and concluded that model should be applied to a single country to reflect its own mortality pattern since mortality patterns by cause-of-death differ by country.

Logistic regression models have been popularly used in actuarial analyses. Especially, De Jong and Heller (2008) and Frees (2010) illustrated that the mortality data, containing binary response (alive or dead) and covariates associated with mortality risk factors, can be fitted suitably by logistic regression models. The model, which is the multinomial logistic regression model, can be extended for multiple responses to ensure that causes-of-death mortality can be suitably modelled by considering various risk factors affecting multiple causes of mortality. Poston and Min (2008) analyzed cause-ofdeath mortality of elderly population in the US using a multinomial logistic regression model. The authors constructed a model for 10 causes of death with several covariates. Alai et al. (2014) observed cause-of-death mortality data in France to quantify the impact of change in mortality due to the main causes of mortality on life expectancy. They suggested that a multinomial logistic model is useful for analyzing cause-of-death mortality as the model is easy and flexible for practical implementations.

\section{Data}

Two sets of data regarding cause-of-death mortality were analyzed in this study to observe the pattern of cause-of-death mortality for different populations. One data-set was obtained from the Centers for Disease Control and Prevention (CDC) in the US. Another data-set was collected from Micro Data Integrated Service (MDIS), administered by Statistics Korea. Both data-sets provide information on population size and the number of deaths by age, sex, and the main causes of deaths for 2000-2016. The main cause of death was coded by the International Code of Disease 10 (ICD-10). In order to construct a reliable mortality model with a reasonable number of deaths for all ages by each cause under consideration, we adopted a classification for causes of death used in Alai et al. (2014) (Table $1)$. 
Table 1: Main causes of death

\begin{tabular}{clcl}
\hline \hline Index & \multicolumn{1}{c}{ Cause of death } & ICD-10 codes & \multicolumn{1}{c}{ Main diseases } \\
\hline 1 & Infectious and parasitic diseases & A00-B99 & $\begin{array}{l}\text { Intestinal infectious disease, Bacterial diseases, } \\
\text { Tuberculosis, Viral hepatitis }\end{array}$ \\
\hline 2 & Cancer & C00-D48 & $\begin{array}{l}\text { Malignant neoplasms, In situ neoplasms, Benign } \\
\text { neoplasms }\end{array}$ \\
\hline 3 & Circulatory system & I00-I99 & $\begin{array}{l}\text { Hypertensive diseases, Ischemic heart diseases, } \\
\text { Acute rheumatic fever }\end{array}$ \\
\hline 4 & Respiratory system & J00-J99 & $\begin{array}{l}\text { Influenza, Pneumonia, Respiratory infections, } \\
\text { Chronic lower respiratory diseases }\end{array}$ \\
\hline 5 & External causes & V00-Y89 & $\begin{array}{l}\text { Accidents, Intentional self-harm, Assault, Com- } \\
\text { plications in medical and surgical care }\end{array}$ \\
\hline \hline
\end{tabular}

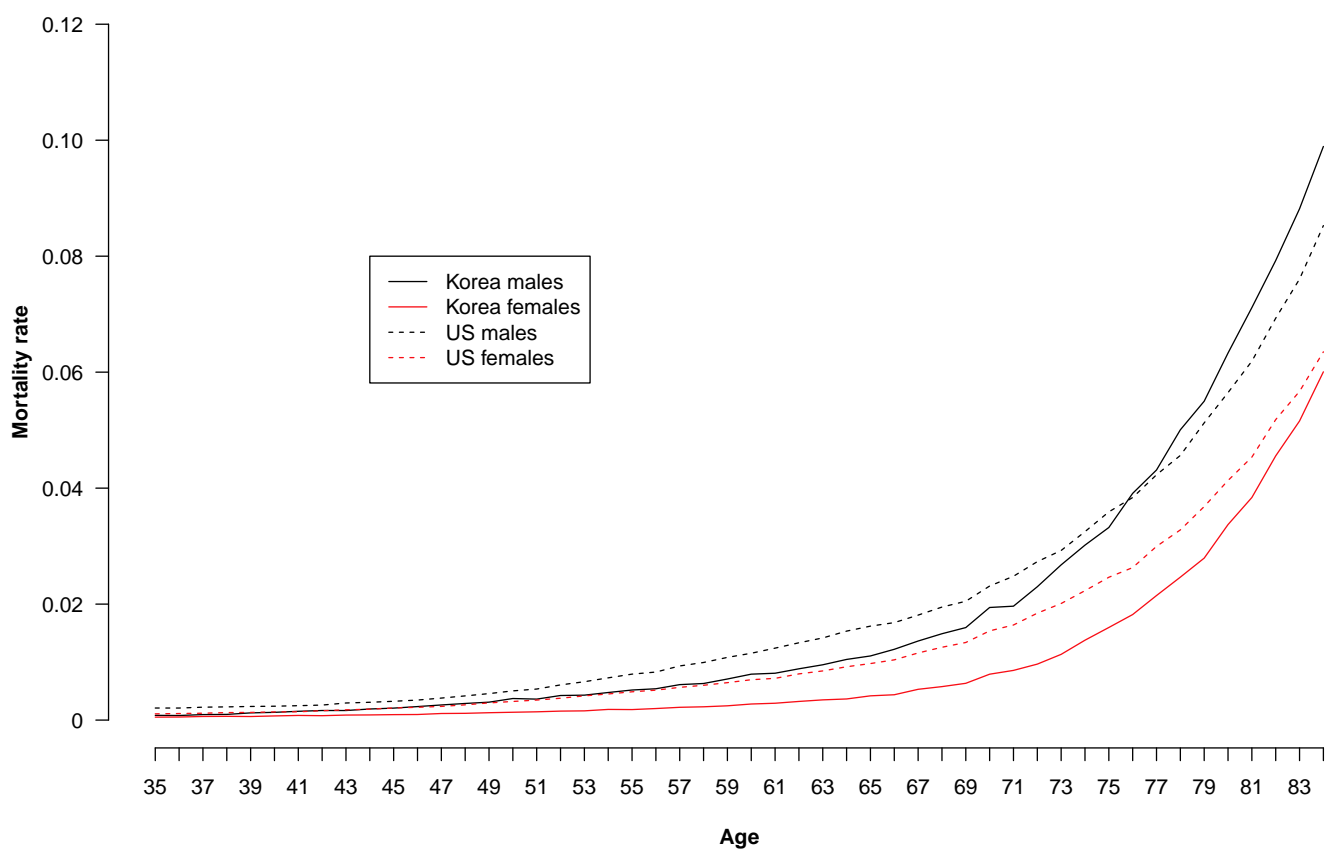

Figure 1: Overall crude mortality in 2016.

Figure 1 shows the overall crude mortality rates over ages 35-84 in 2016. Crude mortality rates follow a typical age-pattern of human mortality and are distinctly different between males and females in each country. However, the mortality gap by sex among the elderly is larger in the Korean population. A comparison of mortality levels between the two countries showed that the mortality level in the Korean population is lower than that in the US population. There is a crossover in the mortality level of males in two countries; in addition, the mortality gap between females in the two countries narrows with increasing age.

Using the cause-of-death data, crude mortality rates in year 2016 by ages and cause of death were observed (Figure 2). Similar to overall mortality, mortality difference between males and females for causes associated with cancer (Cause 2), circulatory system (Cause 3), and respiratory system (Cause 


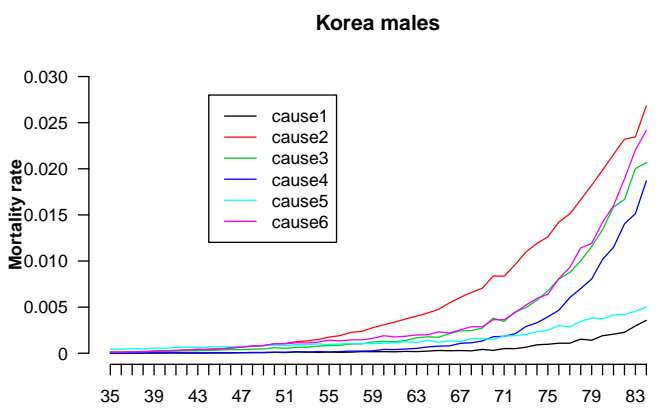

Age

US males

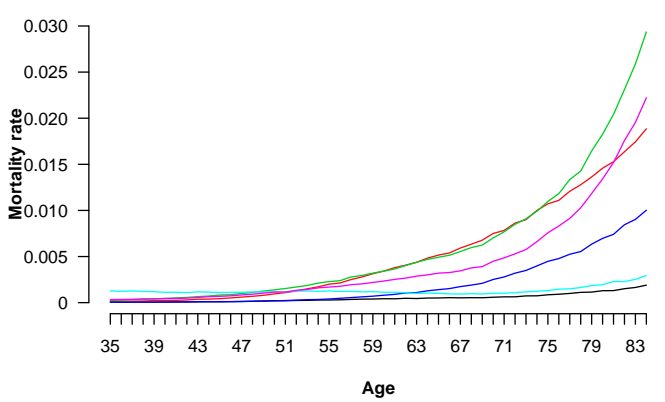

Korea females

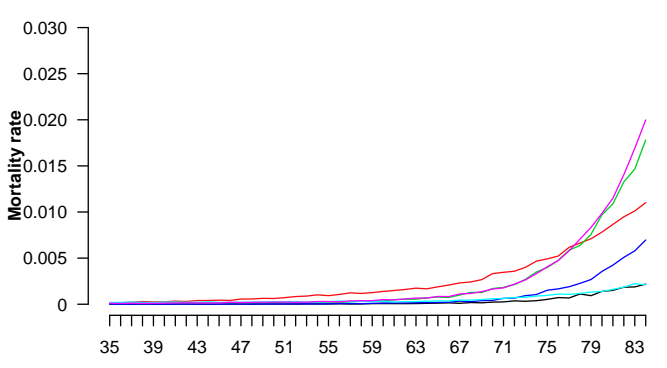

Age

US females

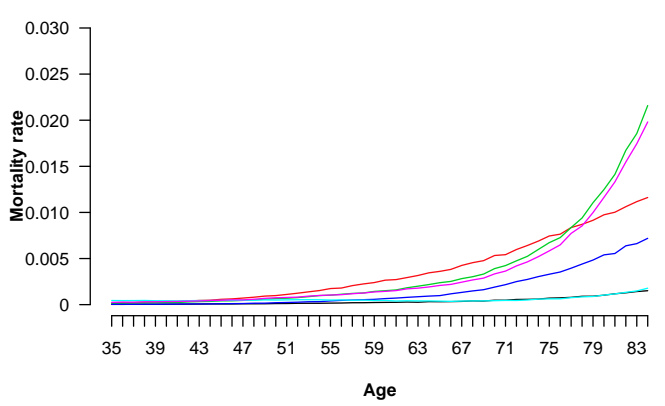

Figure 2: Crude mortality rates by the cause of death in year 2016.

4) were distinct in both countries. Mortality differences between male and females are relatively small for infectious and parasitic diseases (Cause 1) and external causes (Cause 5).

Cancer and circulatory diseases are leading causes of mortality in both sexes, which is consistent with previous findings. In the insurance context, the main coverages of critical illness insurance, such as cancer, stroke, and heart attack, are included in those two categories. From middle ages, males in both countries show steeper increase in mortality due to Cause 2 and Cause 3 . However, the relative age-increasing pattern among causes of mortality differs by country and sex. For example, in case of Korean males, the cancer mortality rate is the highest among the six causes after 50 years. In the case of the US males, mortality due to cancer and the failure of circulatory system are the most popular causes in elderly population aged over 55 years, and similar increasing pattern up to 75 years. Subsequently, a crossover in mortality rates between circulatory system and other causes is observed. The relative increasing pattern in mortality is similar for females in Korea and the US. However, the increasing patterns are different between males and females for both countries. These results suggest that cause-of-death mortality should be analyzed using country-based data.

Mortality improvement can be better understood when trends in mortality rates are separately observed by the cause of death. For example, Figure 3 illustrates crude mortality rates of persons aged between 50 and 59 by from 2000 to 2016 . Mortality improvements in cancer and the circulatory system was observed for males and females in Korea, while the degree of improvement was more distinct in the case of males. The mortality level is expected to stabilize in near future since the mortality level of Korean females is already low. Concerning the population aged 50 years in the 

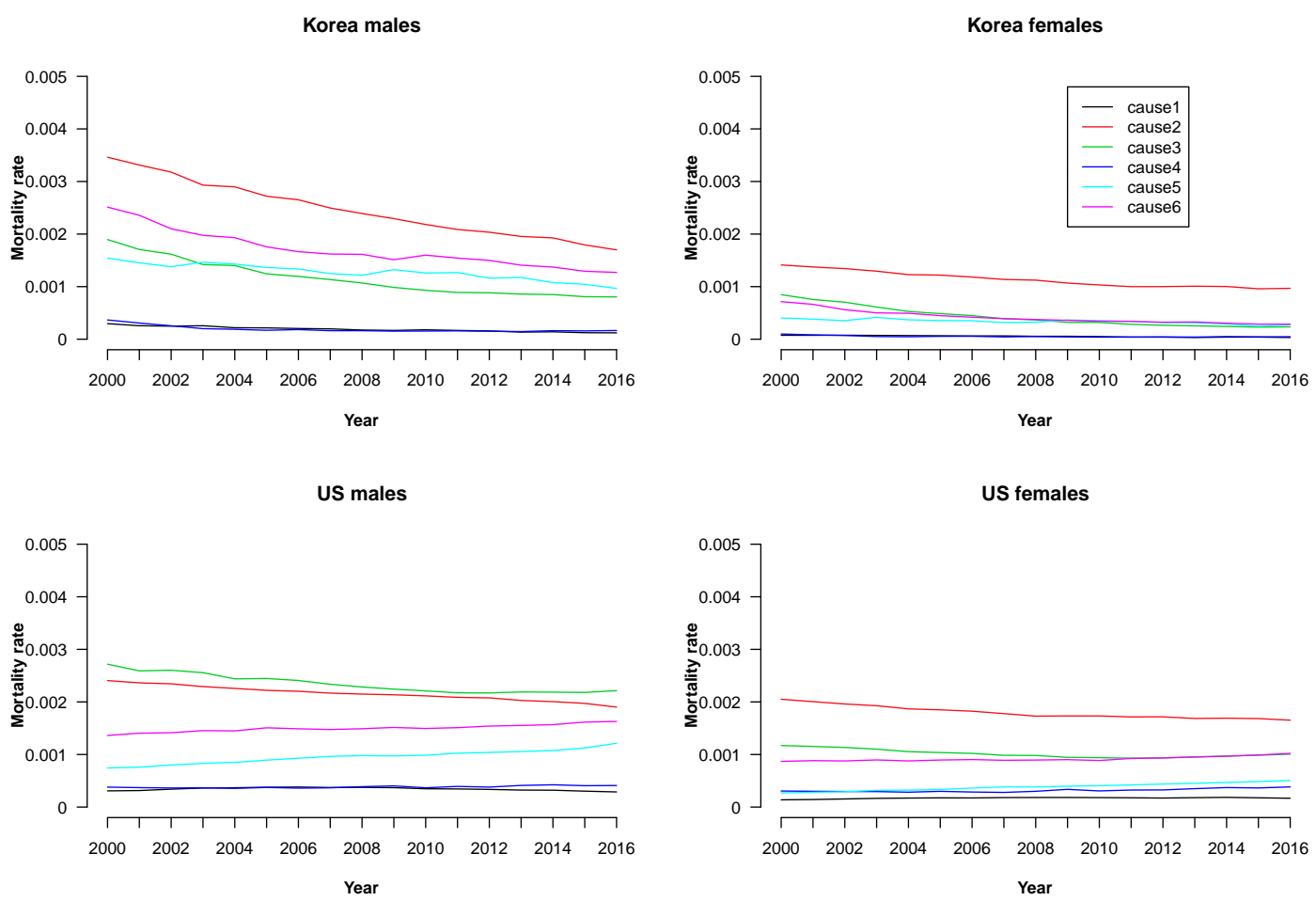

Figure 3: Crude mortality rates of 50's by the cause of death.

US, there are some increasing mortality patterns for Cause 5 and Cause 6. Therefore, the overall mortality improvement should be carefully analyzed as an increasing mortality trend by some causes are overlooked (by the impact of a decreasing trend in some other causes) when only the overall mortality improvement is considered.

\section{Multinomial logistic regression models}

An appropriate statistical model must be constructed to accommodate the causes-of-death mortality under consideration as well as age and year for the actuarial analyses discussed in Section 5. Generalized linear models are popularly used in actuarial modeling, as illustrated in De Jong and Heller (2008) and Frees (2010). Recently, Alai et al. (2014) used a multinomial logistic regression model to analyze the cause of death mortality based on the population data of France. The model is considered suitable for cause-of-death mortality data and easy to implement in actuarial analyses, the mortality data illustrated in the previous section were fitted using the multinomial logistic regression model.

In Alai et al. (2014), the model contained a number of parameters associated with the age covariate because the mortality data were based on 5-year age group. The authors considered age as a categorical variable, which increased the number of parameters in the model. Additionally, the calibration to convert the 5-year age group's mortality rate to a mortality rate for 1-year might induce an additional modelling error. A more parsimonious mortality model could be constructed by considering age as a continuous variable since the data used in this study include mortality experience in each integer age. In addition, smoothing or approximation to convert grouped mortality rate into 
Table 2: Parameters in the selected models (Korea)

\begin{tabular}{|c|c|c|c|c|c|c|c|}
\hline \multirow[b]{2}{*}{ Variable } & \multirow[b]{2}{*}{ Cause } & \multicolumn{3}{|c|}{ Male } & \multicolumn{3}{|c|}{ Female } \\
\hline & & $\begin{array}{l}\text { Estimated } \\
\text { coefficient }\end{array}$ & $\begin{array}{l}\text { Standard } \\
\text { error }\end{array}$ & $p$-value & $\begin{array}{l}\text { Estimated } \\
\text { coefficient }\end{array}$ & $\begin{array}{l}\text { Standard } \\
\text { error }\end{array}$ & $p$-value \\
\hline \multirow{6}{*}{$\beta_{0}$} & 1 & $-9.275 \times 10^{0}$ & $9.415 \times 10^{-2}$ & $<0.0001$ & $-1.063 \times 10^{1}$ & $1.528 \times 10^{-1}$ & $<0.0001$ \\
\hline & 2 & $-1.459 \times 10^{1}$ & $3.447 \times 10^{-2}$ & $<0.0001$ & $-1.093 \times 10^{1}$ & $4.007 \times 10^{-2}$ & $<0.0001$ \\
\hline & 3 & $-1.043 \times 10^{1}$ & $4.083 \times 10^{-2}$ & $<0.0001$ & $-1.169 \times 10^{1}$ & $5.920 \times 10^{-2}$ & $<0.0001$ \\
\hline & 4 & $-1.448 \times 10^{1}$ & $9.976 \times 10^{-2}$ & $<0.0001$ & $-1.214 \times 10^{1}$ & $1.494 \times 10^{-1}$ & $<0.0001$ \\
\hline & 5 & $-7.331 \times 10^{0}$ & $3.756 \times 10^{-2}$ & $<0.0001$ & $-5.559 \times 10^{0}$ & $5.718 \times 10^{-2}$ & $<0.0001$ \\
\hline & 6 & $-7.383 \times 10^{0}$ & $3.270 \times 10^{-2}$ & $<0.0001$ & $-7.461 \times 10^{0}$ & $5.160 \times 10^{-2}$ & $<0.0001$ \\
\hline \multirow{6}{*}{$t$} & 1 & $-9.027 \times 10^{-2}$ & $2.593 \times 10^{-3}$ & $<0.0001$ & $-9.898 \times 10^{-2}$ & $4.302 \times 10^{-3}$ & $<0.0001$ \\
\hline & 2 & $-7.346 \times 10^{-2}$ & $8.432 \times 10^{-4}$ & $<0.0001$ & $-3.305 \times 10^{-2}$ & $1.049 \times 10^{-3}$ & $<0.0001$ \\
\hline & 3 & $-6.501 \times 10^{-2}$ & $1.075 \times 10^{-3}$ & $<0.0001$ & $-1.207 \times 10^{-2}$ & $1.546 \times 10^{-3}$ & $<0.0001$ \\
\hline & 4 & $-8.910 \times 10^{-2}$ & $2.406 \times 10^{-3}$ & $<0.0001$ & $-6.479 \times 10^{-2}$ & $3.811 \times 10^{-3}$ & $<0.0001$ \\
\hline & 5 & $-3.421 \times 10^{-2}$ & $9.729 \times 10^{-4}$ & $<0.0001$ & $-5.060 \times 10^{-2}$ & $1.533 \times 10^{-3}$ & 0.0010 \\
\hline & 6 & $-5.160 \times 10^{-2}$ & $8.873 \times 10^{-4}$ & $<0.0001$ & $-3.759 \times 10^{-2}$ & $1.445 \times 10^{-3}$ & $<0.0001$ \\
\hline \multirow{6}{*}{$x$} & 1 & $-2.775 \times 10^{-2}$ & $3.200 \times 10^{-3}$ & $<0.0001$ & $-3.460 \times 10^{-2}$ & $4.943 \times 10^{-3}$ & $<0.0001$ \\
\hline & 2 & $2.203 \times 10^{-1}$ & $1.119 \times 10^{-3}$ & $<0.0001$ & $7.894 \times 10^{-2}$ & $1.303 \times 10^{-3}$ & $<0.0001$ \\
\hline & 3 & $4.913 \times 10^{-2}$ & $1.316 \times 10^{-3}$ & $<0.0001$ & $5.165 \times 10^{-2}$ & $1.803 \times 10^{-3}$ & $<0.0001$ \\
\hline & 4 & $9.547 \times 10^{-2}$ & $3.036 \times 10^{-3}$ & $<0.0001$ & $-2.707 \times 10^{-2}$ & $4.423 \times 10^{-3}$ & $<0.0001$ \\
\hline & 5 & $-6.415 \times 10^{-3}$ & $1.340 \times 10^{-3}$ & $<0.0001$ & $-1.258 \times 10^{-1}$ & $1.959 \times 10^{-3}$ & $<0.0001$ \\
\hline & 6 & $-3.172 \times 10^{-2}$ & $1.094 \times 10^{-3}$ & $<0.0001$ & $-1.010 \times 10^{-1}$ & $1.586 \times 10^{-3}$ & $<0.0001$ \\
\hline \multirow{6}{*}{$x^{2}$} & 1 & $8.724 \times 10^{-4}$ & $2.699 \times 10^{-5}$ & $<0.0001$ & $1.024 \times 10^{-3}$ & $3.996 \times 10^{-5}$ & $<0.0001$ \\
\hline & 2 & $-1.037 \times 10^{-3}$ & $9.164 \times 10^{-6}$ & $<0.0001$ & $1.256 \times 10^{-5}$ & $1.066 \times 10^{-5}$ & 0.2386 \\
\hline & 3 & $4.781 \times 10^{-4}$ & $1.065 \times 10^{-5}$ & $<0.0001$ & $6.081 \times 10^{-4}$ & $1.381 \times 10^{-5}$ & $<0.0001$ \\
\hline & 4 & $3.777 \times 10^{-4}$ & $2.335 \times 10^{-5}$ & $<0.0001$ & $1.399 \times 10^{-3}$ & $3.324 \times 10^{-5}$ & $<0.0001$ \\
\hline & 5 & $4.076 \times 10^{-4}$ & $1.190 \times 10^{-5}$ & $<0.0001$ & $1.551 \times 10^{-3}$ & $1.654 \times 10^{-5}$ & $<0.0001$ \\
\hline & 6 & $9.907 \times 10^{-4}$ & $9.118 \times 10^{-6}$ & $<0.0001$ & $1.859 \times 10^{-3}$ & $1.229 \times 10^{-5}$ & $<0.0001$ \\
\hline \multirow{6}{*}{$t \times x^{2}$} & 1 & $9.656 \times 10^{-6}$ & $5.553 \times 10^{-7}$ & $<0.0001$ & $1.636 \times 10^{-5}$ & $7.966 \times 10^{-7}$ & $<0.0001$ \\
\hline & 2 & $9.034 \times 10^{-6}$ & $1.799 \times 10^{-7}$ & $<0.0001$ & $2.267 \times 10^{-6}$ & $2.154 \times 10^{-7}$ & $<0.0001$ \\
\hline & 3 & $1.443 \times 10^{-6}$ & $2.170 \times 10^{-7}$ & $<0.0001$ & $8.958 \times 10^{-6}$ & $2.729 \times 10^{-7}$ & $<0.0001$ \\
\hline & 4 & $1.161 \times 10^{-5}$ & $4.369 \times 10^{-7}$ & $<0.0001$ & $5.983 \times 10^{-6}$ & $6.440 \times 10^{-7}$ & $<0.0001$ \\
\hline & 5 & $2.519 \times 10^{-6}$ & $2.549 \times 10^{-7}$ & $<0.0001$ & $-6.146 \times 10^{-6}$ & $3.394 \times 10^{-7}$ & $<0.0001$ \\
\hline & 6 & $1.254 \times 10^{-6}$ & $1.885 \times 10^{-7}$ & $<0.0001$ & $-4.059 \times 10^{-6}$ & $2.536 \times 10^{-7}$ & $<0.0001$ \\
\hline
\end{tabular}

mortality rates for each age for actuarial analyses, which might induce additional modelling error, was unnecessary.

The multinomial logistic regression models should be constructed separately since the mortality patterns are different among the four population groups classified by country and sex. The model framework is described as follows. Let $q_{x, t}^{(j)}$ be the probability that a person aged $x$ dies within a year in year $t$ (the base year is 2000 so that $t=1$ for year 2001) due to cause $j$ corresponding to the index for the cause of death described in Table 1, and $q_{x, t}^{(\tau)}=\sum_{j=1}^{6} q_{x, t}^{(j)}$. Further, denote the probability that a person aged $x$ survives one year in year $t$ by $p_{x, t}^{(\tau)}$ so that $p_{x, t}^{(\tau)}=1-q_{x, t}^{(\tau)}$. Then, the multinomial logistic regression model is expressed as:

$$
\ln \frac{q_{x, t}^{(j)}}{p_{x, t}^{(\tau)}}=\beta_{0}^{(j)}+\left(\underline{\beta}^{(j)}\right)^{\prime} \underline{y}, \quad \text { for } j=1,2, \ldots, 6
$$

where $\underline{y}$ is the column vector of variables and their interactions included in the model, $\underline{\beta}^{(j)}$ is the column vector of the coefficients corresponding to $y$ for mortality due to cause $j$, and $\bar{\beta}_{0}^{(j)}$ is the constant term of the model for mortality due to cause $\bar{j}$. 
Table 3: Parameters in the selected models (US)

\begin{tabular}{|c|c|c|c|c|c|c|c|}
\hline \multirow[b]{2}{*}{ Variable } & \multirow[b]{2}{*}{ Cause } & \multicolumn{3}{|c|}{ Male } & \multicolumn{3}{|c|}{ Female } \\
\hline & & $\begin{array}{l}\text { Estimated } \\
\text { coefficient }\end{array}$ & $\begin{array}{c}\text { Standard } \\
\text { error }\end{array}$ & $p$-value & $\begin{array}{l}\text { Estimated } \\
\text { coefficient }\end{array}$ & $\begin{array}{l}\text { Standard } \\
\text { error }\end{array}$ & $p$-value \\
\hline \multirow{6}{*}{$\beta_{0}$} & 1 & $-2.076 \times 10^{1}$ & $1.374 \times 10^{-1}$ & $<0.0001$ & $-1.311 \times 10 ?^{1}$ & $1.614 \times 10^{-1}$ & $<0.0001$ \\
\hline & 2 & $-1.949 \times 10^{1}$ & $7.000 \times 10^{-2}$ & $<0.0001$ & $-1.505 \times 10^{1}$ & $6.377 \times 10^{-2}$ & $<0.0001$ \\
\hline & 3 & $-2.024 \times 10^{1}$ & $5.805 \times 10^{-2}$ & $<0.0001$ & $-1.636 \times 10^{1}$ & $7.245 \times 10^{-2}$ & $<0.0001$ \\
\hline & 4 & $-1.287 \times 10^{1}$ & $1.350 \times 10^{-1}$ & $<0.0001$ & $-1.230 \times 10^{1}$ & $1.358 \times 10^{-1}$ & $<0.0001$ \\
\hline & 5 & $-1.511 \times 10^{1}$ & $7.280 \times 10^{-2}$ & $<0.0001$ & $-1.872 \times 10^{1}$ & $1.052 \times 10^{-1}$ & $<0.0001$ \\
\hline & 6 & $-1.890 \times 10^{1}$ & $6.558 \times 10^{-2}$ & $<0.0001$ & $-1.456 \times 10^{1}$ & $7.085 \times 10^{-3}$ & $<0.0001$ \\
\hline \multirow{6}{*}{$t$} & 1 & $-2.617 \times 10^{-1}$ & $2.466 \times 10^{-3}$ & $<0.0001$ & $-1.318 \times 10^{-1}$ & $2.737 \times 10^{-3}$ & $<0.0001$ \\
\hline & 2 & $-1.642 \times 10^{-2}$ & $1.220 \times 10^{-3}$ & $<0.0001$ & $-2.538 \times 10^{-3}$ & $1.069 \times 10^{-3}$ & 0.0297 \\
\hline & 3 & $8.200 \times 10^{-3}$ & $1.019 \times 10^{-3}$ & $<0.0001$ & $3.412 \times 10^{-2}$ & $1.221 \times 10^{-3}$ & $<0.0001$ \\
\hline & 4 & $2.858 \times 10^{-3}$ & $2.445 \times 10^{-3}$ & 0.2425 & $2.978 \times 10^{-2}$ & $2.327 \times 10^{-3}$ & $<0.0001$ \\
\hline & 5 & $-2.547 \times 10^{-2}$ & $1.208 \times 10^{-3}$ & $<0.0001$ & $-3.618 \times 10^{-4}$ & $1.669 \times 10^{-3}$ & 0.0458 \\
\hline & 6 & $-3.758 \times 10^{-2}$ & $1.164 \times 10^{-3}$ & $<0.0001$ & $1.629 \times 10^{-2}$ & $1.200 \times 10^{-3}$ & $<0.0001$ \\
\hline \multirow{6}{*}{$x$} & 1 & $7.080 \times 10^{-1}$ & $7.290 \times 10^{-3}$ & $<0.0001$ & $2.125 \times 10^{-1}$ & $8.350 \times 10^{-3}$ & $<0.0001$ \\
\hline & 2 & $4.227 \times 10^{-1}$ & $3.427 \times 10^{-3}$ & $<0.0001$ & $2.363 \times 10^{-1}$ & $3.159 \times 10^{-3}$ & $<0.0001$ \\
\hline & 3 & $5.530 \times 10^{-1}$ & $2.857 \times 10^{-3}$ & $<0.0001$ & $3.148 \times 10^{-1}$ & $3.496 \times 10^{-3}$ & $<0.0001$ \\
\hline & 4 & $1.233 \times 10^{-2}$ & $6.496 \times 10^{-3}$ & 0.0577 & $-2.786 \times 10^{-2}$ & $6.502 \times 10^{-3}$ & $<0.0001$ \\
\hline & 5 & $4.978 \times 10^{-1}$ & $3.945 \times 10^{-3}$ & $<0.0001$ & $6.331 \times 10^{-1}$ & $5.583 \times 10^{-3}$ & $<0.0001$ \\
\hline & 6 & $5.482 \times 10^{-1}$ & $3.319 \times 10^{-3}$ & $<0.0001$ & $2.838 \times 10^{-1}$ & $3.521 \times 10^{-3}$ & $<0.0001$ \\
\hline \multirow{6}{*}{$x^{2}$} & 1 & $-1.297 \times 10^{-2}$ & $1.266 \times 10^{-4}$ & $<0.0001$ & $-3.920 \times 10^{-3}$ & $1.421 \times 10^{-4}$ & $<0.0001$ \\
\hline & 2 & $-4.007 \times 10^{-3}$ & $5.569 \times 10^{-5}$ & $<0.0001$ & $-1.457 \times 10^{-3}$ & $5.203 \times 10^{-5}$ & $<0.0001$ \\
\hline & 3 & $-7.541 \times 10^{-3}$ & $4.655 \times 10^{-5}$ & $<0.0001$ & $-3.742 \times 10^{-3}$ & $5.602 \times 10^{-5}$ & $<0.0001$ \\
\hline & 4 & $2.131 \times 10^{-3}$ & $1.038 \times 10^{-4}$ & $<0.0001$ & $2.876 \times 10^{-3}$ & $1.039 \times 10^{-4}$ & $<0.0001$ \\
\hline & 5 & $-9.909 \times 10^{-3}$ & $7.025 \times 10^{-5}$ & $<0.0001$ & $-1.225 \times 10^{-2}$ & $9.784 \times 10^{-5}$ & $<0.0001$ \\
\hline & 6 & $-8.687 \times 10^{-3}$ & $5.547 \times 10^{-5}$ & $<0.0001$ & $-4.129 \times 10^{-3}$ & $5.807 \times 10^{-5}$ & $<0.0001$ \\
\hline \multirow{6}{*}{$x^{3}$} & 1 & $7.924 \times 10^{-5}$ & $7.170 \times 10^{-7}$ & $<0.0001$ & $2.812 \times 10^{-5}$ & $7.932 \times 10^{-7}$ & $<0.0001$ \\
\hline & 2 & $1.455 \times 10^{-5}$ & $2.990 \times 10^{-7}$ & $<0.0001$ & $2.088 \times 10^{-6}$ & $2.833 \times 10^{-7}$ & $<0.0001$ \\
\hline & 3 & $4.074 \times 10^{-5}$ & $2.501 \times 10^{-7}$ & $<0.0001$ & $2.223 \times 10^{-5}$ & $2.968 \times 10^{-7}$ & $<0.0001$ \\
\hline & 4 & $-1.242 \times 10^{-5}$ & $5.485 \times 10^{-7}$ & $<0.0001$ & $-1.748 \times 10^{-5}$ & $5.500 \times 10^{-7}$ & $<0.0001$ \\
\hline & 5 & $6.346 \times 10^{-5}$ & $4.090 \times 10^{-7}$ & $<0.0001$ & $7.724 \times 10^{-5}$ & $5.626 \times 10^{-7}$ & $<0.0001$ \\
\hline & 6 & $5.130 \times 10^{-5}$ & $3.048 \times 10^{-7}$ & $<0.0001$ & $2.680 \times 10^{-5}$ & $3.159 \times 10^{-7}$ & $<0.0001$ \\
\hline \multirow{6}{*}{$t \times x^{2}$} & 1 & $1.736 \times 10^{-4}$ & $1.943 \times 10^{-6}$ & $<0.0001$ & $8.776 \times 10^{-5}$ & $2.059 \times 10^{-6}$ & $<0.0001$ \\
\hline & 2 & $-3.031 \times 10^{-6}$ & $8.422 \times 10^{-7}$ & 0.0003 & $-1.406 \times 10^{-5}$ & $7.545 \times 10^{-7}$ & $<0.0001$ \\
\hline & 3 & $-1.247 \times 10^{-5}$ & $7.165 \times 10^{-7}$ & $<0.0001$ & $-3.165 \times 10^{-5}$ & $8.188 \times 10^{-7}$ & $<0.0001$ \\
\hline & 4 & $2.421 \times 10^{-6}$ & $1.597 \times 10^{-6}$ & 0.1294 & $-1.550 \times 10^{-5}$ & $1.511 \times 10^{-6}$ & $<0.0001$ \\
\hline & 5 & $3.951 \times 10^{-5}$ & $1.092 \times 10^{-6}$ & $<0.0001$ & $2.703 \times 10^{-5}$ & $1.446 \times 10^{-6}$ & $<0.0001$ \\
\hline & 6 & $2.610 \times 10^{-5}$ & $8.536 \times 10^{-7}$ & $<0.0001$ & $-1.378 \times 10^{-5}$ & $8.466 \times 10^{-7}$ & $<0.0001$ \\
\hline \multirow{6}{*}{$t \times x^{3}$} & 1 & $-1.667 \times 10^{-6}$ & $2.007 \times 10^{-8}$ & $<0.0001$ & $-8.345 \times 10^{-7}$ & $2.089 \times 10^{-8}$ & $<0.0001$ \\
\hline & 2 & $3.281 \times 10^{-8}$ & $8.338 \times 10^{-9}$ & $<0.0001$ & $1.588 \times 10^{-7}$ & $7.526 \times 10^{-9}$ & $<0.0001$ \\
\hline & 3 & $6.243 \times 10^{-8}$ & $7.112 \times 10^{-9}$ & $<0.0001$ & $2.503 \times 10^{-7}$ & $7.968 \times 10^{-9}$ & $<0.0001$ \\
\hline & 4 & $-7.395 \times 10^{-8}$ & $1.544 \times 10^{-8}$ & $<0.0001$ & $1.204 \times 10^{-7}$ & $1.456 \times 10^{-8}$ & $<0.0001$ \\
\hline & 5 & $-4.487 \times 10^{-7}$ & $1.182 \times 10^{-8}$ & $<0.0001$ & $-3.274 \times 10^{-7}$ & $1.528 \times 10^{-8}$ & $<0.0001$ \\
\hline & 6 & $-2.409 \times 10^{-7}$ & $8.584 \times 10^{-9}$ & $<0.0001$ & $1.551 \times 10^{-7}$ & $8.384 \times 10^{-9}$ & $<0.0001$ \\
\hline
\end{tabular}

Age, denoted by $x$ and year, denoted by $t$, and their interactions were considered as explanatory variables in the model. Mortality data based on population ages over 35 years were used for model construction since the mortality differential by the cause of death becomes more significant in older ages. After exploring possible models with various functional forms of explanatory variables and its interactions, the final model was selected based on the number of parameters together with the Akaike Information Criterion (AIC). Tables 2 and 3 presents the explanatory variables in the finally 

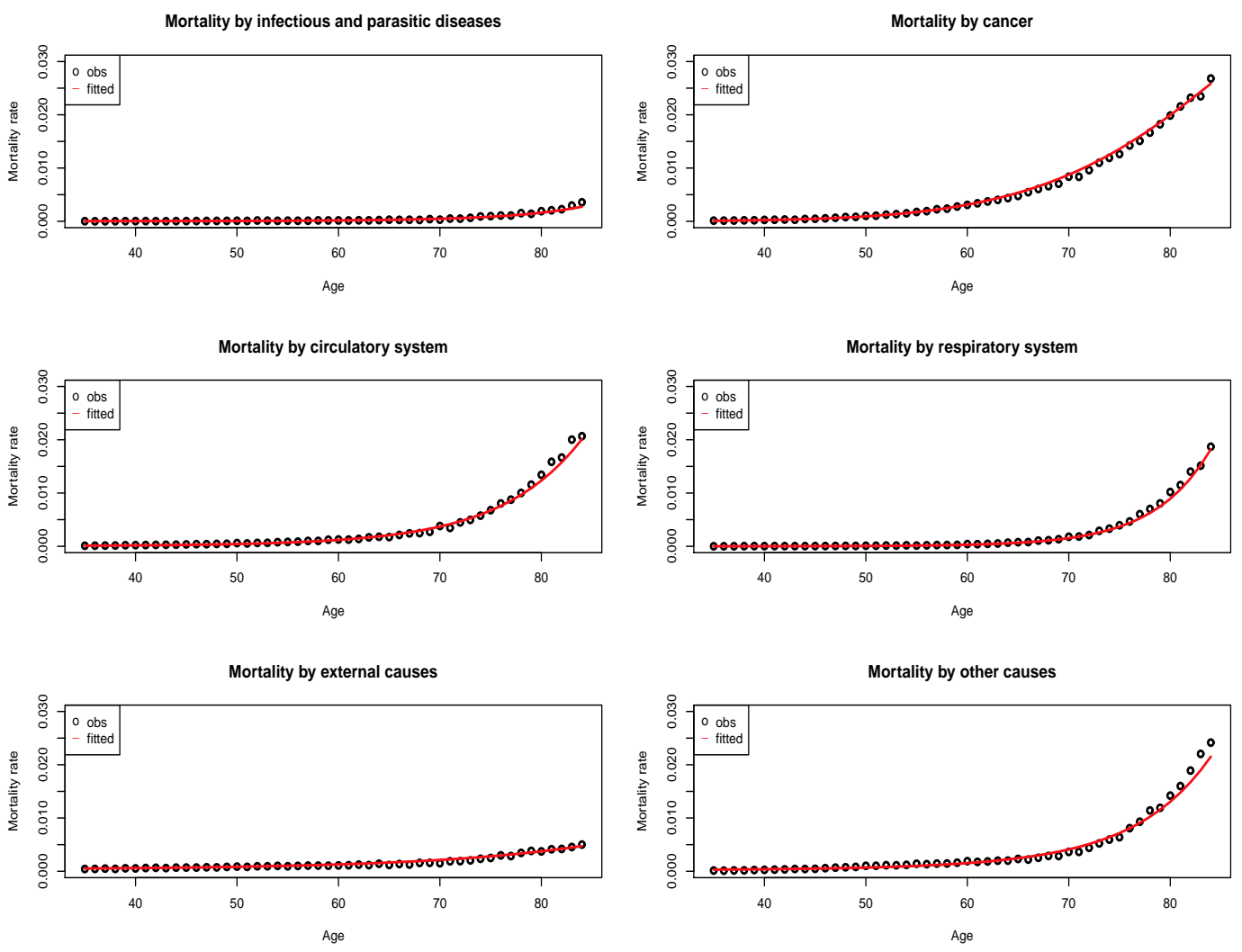

Figure 4: Goodness-of-fit of the multinomial logistic regression model (Korean male).

selected models and their estimated coefficients, together with their significance for Korean and US populations. Figures 4 and 5 shows the goodness of fit of the selected models.

Multinomial generalized linear models fit age-pattern and the trend of the cause-of-death mortality fairly well even when unabridged mortality data is used. Further, functional forms of the model are different between the two populations. This result is attributed to the difference in age-increasing patterns and trends discussed in Section 2. In addition, a model combining males and females was not significant because the age-increasing pattern and trend in the cause-of-death mortality are different by sex with a mortality gap that changes by age.

\section{Actuarial analyses}

The impact of mortality improvement due to a certain cause or multiple causes on actuarial values can be quantified based on the developed of the multinomial logistic regression models in the previous section. These results can be used to evaluate mortality/longevity risks. The implementation of actuarial analysis is straightforward and based on the framework of the multiple decrement model since the models consider age and time as continuous variables. When the impact of full or partial elimination of mortality due to cause $j$ is to be evaluated, adjustments for mortality rates due to causes other than cause $j$, together with survival probability, will be necessary since the sum of mortality rates for all causes and survival probability must still be 1 . 

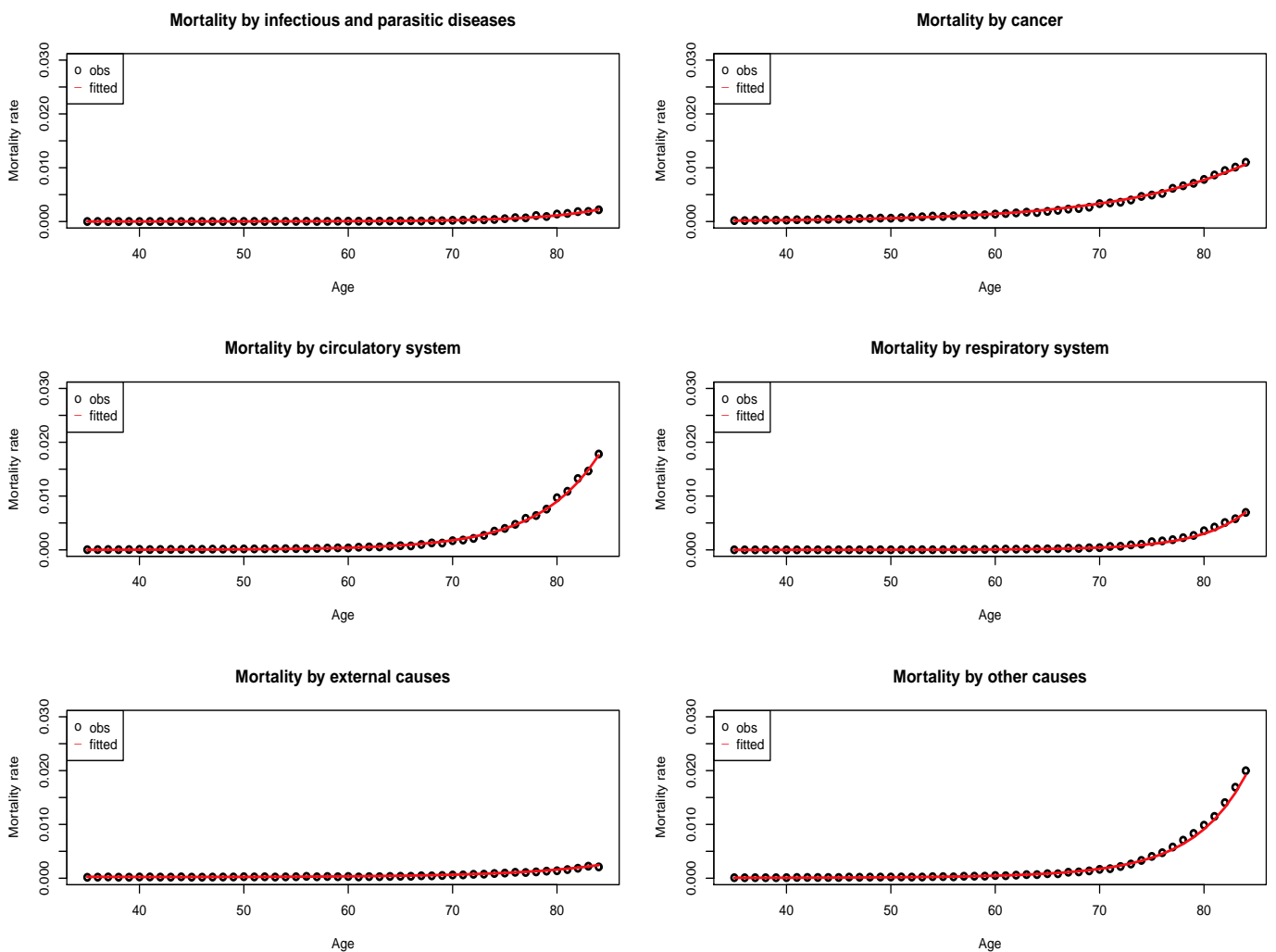

Figure 5: Goodness-of-fit of the multinomial logistic regression model (Korean female).

Consider that the impact of mortality improvement in cause $j$ is to be evaluated. Let current probability that a person aged $x$ in year $t$ will die due to cause $j$ be denoted by $q_{x, t}^{(j)}$. If mortality rates due to cause $j$ become $100(1-\alpha) \%$ of the previous levels where $\alpha$ indicates the degree of mortality reduction, then mortality rate due to cause $j$ for age $x$ in year $t$ is $q_{x, t}^{*(j)}=(1-\alpha) \cdot q_{x, t}^{(j)}$, where $q_{x, t}^{*(j)}$ is updated mortality rate due to cause $j$ for age $x$ in year $t$. Therefore, the value of $\alpha$ indicates how much the level of mortality due to cause $j$ differs from the expected mortality rate due to cause $j$ based on mortality model specified in the previous section. For example, if the value of $\alpha$ is positive, the mortality level is lower than expected by the specified model. However, the negative value of $\alpha$ represents a higher mortality level compared with mortality rate derived by the specified model.

As a result, the extra change in amount of mortality $\alpha \cdot q_{x, t}^{(j)}$ must be distributed and added to $q_{x, t}^{(k)}$ for each cause $k(\neq j)$ and survival probability. Information on the dependence structure among causes is not available; therefore, we assigned the reduced mortality in cause $j$ to other probabilities proportionally, as illustrated in Alai et al. (2014). That is,

$$
\begin{aligned}
& q_{x, t}^{*(k)}=q_{x, t}^{(k)}+\alpha \cdot q_{x, t}^{(j)} \cdot \frac{q_{x, t}^{(k)}}{1-q_{x, t}^{(j)}}, \quad \text { for } k \neq j, \\
& p_{x, t}^{*(\tau)}=p_{x, t}^{(\tau)}+\alpha \cdot q_{x, t}^{(j)} \cdot \frac{p_{x, t}^{(\tau)}}{1-q_{x, t}^{(j)}},
\end{aligned}
$$


Table 4: Effect of cause elimination on the expected 20-year term curtate lifetime

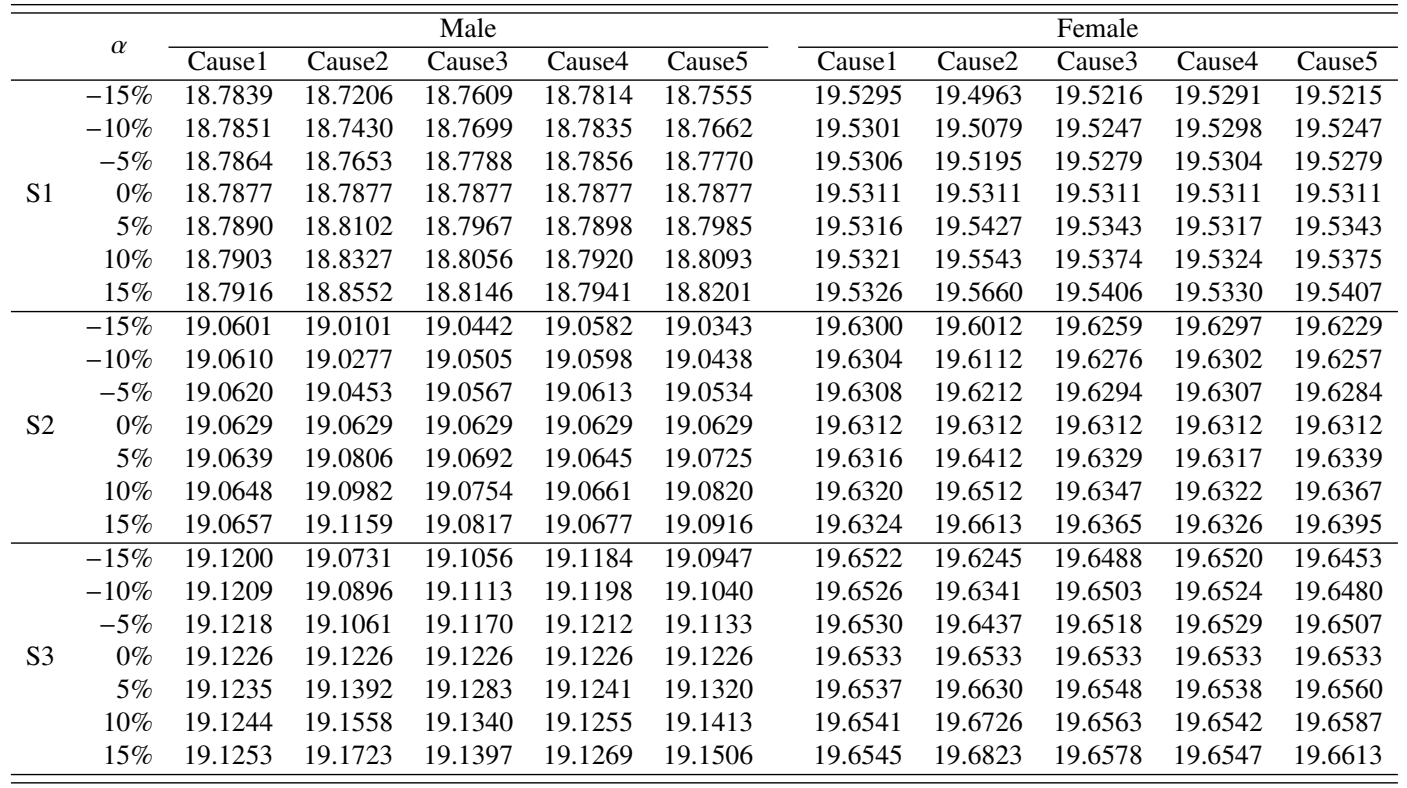

where $p_{x, t}^{(\tau)}$ is probability that a person aged $x$ in year $t$ survives one year and $p_{x, t}^{*(\tau)}$ is the adjusted survival probability reflecting mortality reduction due to a cause. Based on equations (5.1) and (5.2), the impact of mortality improvement in cause $j$ on life expectancy and actuarial values were quantified. The constructed mortality model captures the past trend of mortality rates by year; however, it is uncertain how long the modelled mortality trend will continue in the future. Therefore, the three scenarios regarding future mortality trend were considered to understand how the estimated values are changed according to variations in the mortality trend. In Scenario 1 (S1), it was assumed that the future morality level follows the most recent level of mortality without reflecting any trend. In Scenario 2 (S2), the past trend will continue only for the next 10 years and the mortality level will not change. Finally, in Scenario 3 (S3), the modelled mortality trend will continue in the future.

Based on the three scenarios, the effect of mortality shock due to a specific cause, represented by $\alpha$, was observed. Recent developments in the insurance accounting standard includes the evaluation of mortality/longevity risk based on the analysis of effect of mortality shock, which is the variation of the level of mortality from the best estimate, on the actuarial values. Solvency II regulation documented by the European Insurance and Occupational Pension Authority (2014) specifies 15\% of mortality shock to determine the capital requirement for mortality risk. Additionally, the first draft of the International Financial Reporting Standard 17 (IFRS 17) regulates the principles for evaluation of non-financial risk. Therefore, actuarial values corresponding to various values of $\alpha$ including $15 \%$ were calculated.

First, the expected 20-year term curtate lifetime of a person aged 50 years was calculated, with some modification of the formula illustrated in Dickson, et al. (2013), by

$$
\sum_{k=1}^{20}{ }_{k} p_{50+j, 2016+j}^{*(\tau)}, \quad \text { where }{ }_{k} p_{50+j, 2016+j}^{*(\tau)}= \begin{cases}1, & k=0, \\ \prod_{j=0}^{k-1} p_{50+j, 2016+j}^{*(\tau)}, & k=1,2, \ldots\end{cases}
$$

Table 4 summarizes the results. For life expectancy, both positive and negative values of $\alpha$ ranging 
from $-15 \%$ to $15 \%$ were considered. As expected, the effect of deviation of mortality level, which may be due to faster or slower speed of mortality improvement than expected; however, the effect of cancer on life expectancy is the largest for both sexes. It was observed that the effects are likely to be larger for males than females. If some diseases are associated, such as complications according to a certain disease, the effect of mortality reduction will also extend. For example, if the incidence rate of diabetes is reduced due to the development of medical innovation, then the cause of death mortality associated with diabetic complications will decrease simultaneously. It is expected that the impact of deviation of mortality level from the best estimate on life expectancy will be larger as the considered age increases since the mortality level by main causes becomes larger by age.

It is essential to evaluate the effect of mortality shock on actuarial values, based on the developed mortality model. The actuarial present values were calculated for a person aged 50 years for a 20 -year term life insurance policy payable at a fixed amount at the end of the year of death and for a 20-year term life annuity contract payable at the beginning of each year. The assumed annual effective rate of interest was 3\%. Based on the constructed model, the actuarial present value for the life policy is expressed by,

$$
\sum_{k=0}^{19}(1.03)^{-k-1} \cdot{ }_{k} p_{50+j, 2016+j}^{*(\tau)} \cdot q_{50+k, 2016+k}^{*(k)},
$$

and the actuarial present value for the annuity policy is calculated by,

$$
\sum_{k=0}^{19}(1.03)^{-k-1} \cdot{ }_{k} p_{50+j, 2016+j}^{*(\tau)}
$$

For the case of 20-year term life insurance policy, risk is associated with a higher mortality than expected. Mortality risk should be evaluated by considering the negative values of $\alpha$. Actuarial present values were calculated with values of $\alpha$ that range from $0 \%$ to $-25 \%$ for each cause of death. However, a 20-year term annuity policy is exposed to a longevity risk that is represented by positive values of $\alpha$. Therefore, actuarial present values of annuity were observed by considering positive values of $\alpha$ that range from $0 \%$ to $25 \%$. For all cases, a $15 \%$ shock were included, as guided by Solvency II regulation.

Tables 5 and 6 and presents the calculated actuarial values by the three scenarios and various values of $\alpha$. For comparison, the actuarial present value for the case of Scenario 1 and $\alpha=0$ was set to 1 . For life insurance policy, the impact of the variation of mortality level on actuarial value varies by cause of death. Based on $-15 \%$ mortality shock in Scenario 1, for males, actuarial value increased by $5.59 \%$ for Cause 2, $2.24 \%$ for Cause 5, and $2.23 \%$ for Cause 3 . For females, the actuarial value increased by $6.82 \%$ for Cause $2,2.87 \%$ for Cause 3 , and $1.87 \%$ for Cause 5 . If the impact is observed by scenario focusing on Cause 2, for males, actuarial value increased by $5.59 \%$ in Scenario $1,5.83 \%$ in Scenario 2, and 5.91\%. For females, actuarial value increased by $6.82 \%$ in Scenario $1,7.58 \%$ in Scenario 2, and $7.91 \%$ in Scenario 3. The results imply that mortality risk analysis considering main causes of mortality can provide useful insights. It is more reasonable to consider various scenarios on mortality trends since the mortality trend reflected in the model significantly affects the analysis results.

Longevity risk was quantified in annuity policy. Based on $15 \%$ mortality shock in Scenario 1 , for males, actuarial value increased by $0.27 \%$ for Cause $2,0.13 \%$ for Cause 5 , and $0.11 \%$ for Cause 3 . For females, actuarial value increased by $0.15 \%$ for Cause $2,0.05 \%$ for Cause 5 , and $0.04 \%$ for Cause 
Table 5: Effect of cause elimination on the actuarial present value of the 20-year term life insurance

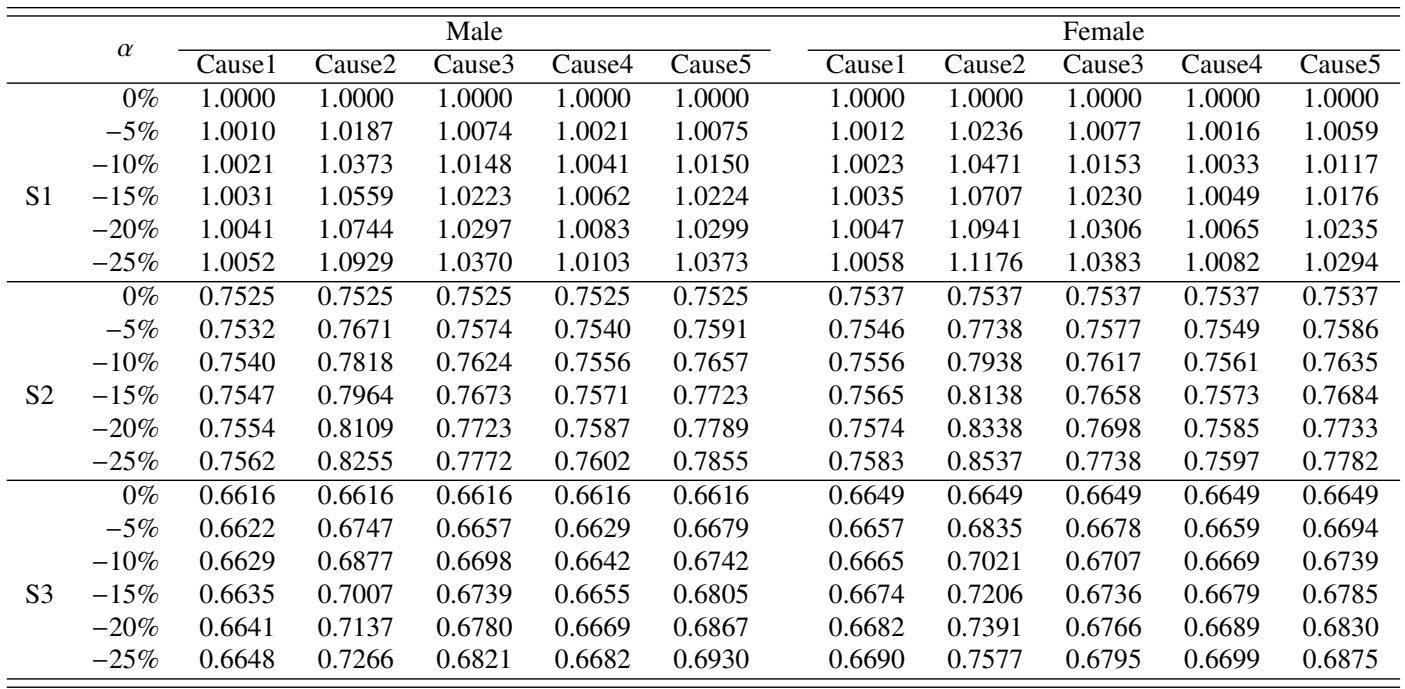

Table 6: Effect of cause elimination on the actuarial present value of the 20-year term life annuity

\begin{tabular}{|c|c|c|c|c|c|c|c|c|c|c|c|}
\hline & \multirow[b]{2}{*}{$\alpha$} & \multicolumn{5}{|c|}{ Male } & \multicolumn{5}{|c|}{ Female } \\
\hline & & Cause1 & Cause 2 & Cause 3 & Cause 4 & Cause 5 & Cause1 & Cause2 & Cause3 & Cause4 & Cause5 \\
\hline \multirow{6}{*}{$\mathrm{S} 1$} & $0 \%$ & 1.0000 & 1.0000 & 1.0000 & 1.0000 & 1.0000 & 1.0000 & 1.0000 & 1.0000 & 1.0000 & 1.0000 \\
\hline & $5 \%$ & 1.0001 & 1.0009 & 1.0004 & 1.0001 & 1.0004 & 1.0000 & 1.0005 & 1.0001 & 1.0000 & 1.0001 \\
\hline & $10 \%$ & 1.0001 & 1.0018 & 1.0007 & 1.0002 & 1.0009 & 1.0000 & 1.0009 & 1.0002 & 1.0000 & 1.0003 \\
\hline & $15 \%$ & 1.0002 & 1.0027 & 1.0011 & 1.0002 & 1.0013 & 1.0001 & 1.0014 & 1.0003 & 1.0001 & 1.0004 \\
\hline & $20 \%$ & 1.0002 & 1.0035 & 1.0014 & 1.0003 & 1.0018 & 1.0001 & 1.0018 & 1.0005 & 1.0001 & 1.0005 \\
\hline & $25 \%$ & 1.0003 & 1.0044 & 1.0018 & 1.0004 & 1.0023 & 1.0001 & 1.0023 & 1.0006 & 1.0001 & 1.0006 \\
\hline \multirow{6}{*}{$\mathrm{S} 2$} & $0 \%$ & 1.0104 & 1.0104 & 1.0104 & 1.0104 & 1.0104 & 1.0036 & 1.0036 & 1.0036 & 1.0036 & 1.0036 \\
\hline & $5 \%$ & 1.0104 & 1.0111 & 1.0107 & 1.0105 & 1.0108 & 1.0036 & 1.0040 & 1.0037 & 1.0036 & 1.0037 \\
\hline & $10 \%$ & 1.0105 & 1.0118 & 1.0109 & 1.0105 & 1.0112 & 1.0036 & 1.0044 & 1.0037 & 1.0036 & 1.0038 \\
\hline & $15 \%$ & 1.0105 & 1.0125 & 1.0112 & 1.0106 & 1.0116 & 1.0036 & 1.0048 & 1.0038 & 1.0036 & 1.0039 \\
\hline & $20 \%$ & 1.0106 & 1.0132 & 1.0114 & 1.0106 & 1.0120 & 1.0037 & 1.0052 & 1.0039 & 1.0037 & 1.0040 \\
\hline & $25 \%$ & 1.0106 & 1.0139 & 1.0117 & 1.0107 & 1.0124 & 1.0037 & 1.0056 & 1.0039 & 1.0037 & 1.0042 \\
\hline \multirow{6}{*}{ S3 } & $0 \%$ & 1.0122 & 1.0122 & 1.0122 & 1.0122 & 1.0122 & 1.0042 & 1.0042 & 1.0042 & 1.0042 & 1.0042 \\
\hline & $5 \%$ & 1.0122 & 1.0129 & 1.0124 & 1.0123 & 1.0126 & 1.0042 & 1.0046 & 1.0043 & 1.0042 & 1.0043 \\
\hline & $10 \%$ & 1.0123 & 1.0135 & 1.0127 & 1.0123 & 1.0130 & 1.0043 & 1.0050 & 1.0043 & 1.0043 & 1.0044 \\
\hline & $15 \%$ & 1.0123 & 1.0142 & 1.0129 & 1.0124 & 1.0134 & 1.0043 & 1.0054 & 1.0044 & 1.0043 & 1.0046 \\
\hline & $20 \%$ & 1.0123 & 1.0149 & 1.0131 & 1.0124 & 1.0138 & 1.0043 & 1.0058 & 1.0045 & 1.0043 & 1.0047 \\
\hline & $25 \%$ & 1.0124 & 1.0155 & 1.0134 & 1.0125 & 1.0142 & 1.0043 & 1.0061 & 1.0045 & 1.0043 & 1.0048 \\
\hline
\end{tabular}

3. If the impact is observed by Scenario focusing on Cause 2, for males, actuarial value increased by $0.27 \%$ in Scenario $1,0.20 \%$ in Scenario 2, and $0.20 \%$ in Scenario 3. For females, actuarial value increased by $0.15 \%$ in Scenario $1,0.14 \%$ in Scenario 2, and $0.13 \%$ in Scenario 3. The impact of variation of mortality by cause is relatively smaller in annuity policy; in addition, the impact does not vary across scenarios of mortality trend.

As expected, the variation of mortality due to Cause 2 significantly affects the actuarial present values. The change in actuarial value of annuity policy expands if the impact of mortality reduction due to Cause 2 is combined with Scenario 3, which is the worst scenario from the perspective of longevity risk. This implies that the impact of mortality reduction due to some major causes on 


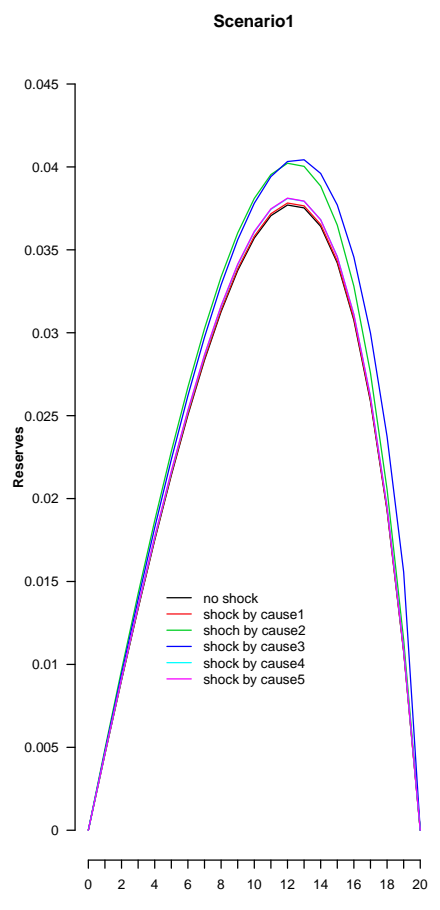

Duratio
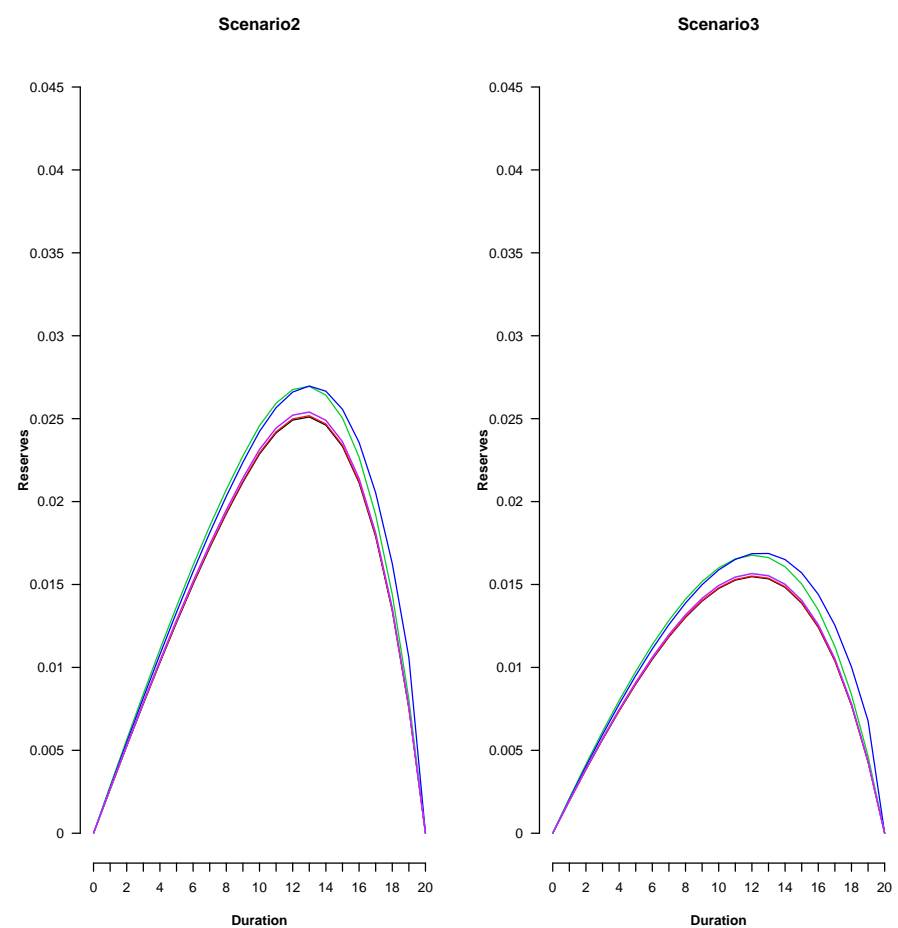

Figure 6: Net premium reserve by duration for 20-year term life insurance (male).

longevity risk should be closely analyzed and reflected in actuarial risk management. The model will be useful in actuarial practice since the actuarial analysis of cause-of-death mortality, based on multinomial logistic regression model is as illustrated.

The variation of net premium reserve by duration for the illustrated life insurance policy was observed according to the $-15 \%$ mortality shock by each cause of death. The net premiums were calculated by equivalence principle. Figures 6 and 7 presents the results. It was observed that the reserve level decreases as mortality trends last longer. Additionally, the variations of reserve level diminish as mortality trends last longer. These results reflect that the reserve decreases as mortality improvement reduces mortality.

\section{Concluding remarks}

Previous studies on cause-of-death mortality have provide useful insights to understand important aspects of human mortality. Especially, an analysis of cause-of-death mortality can be associated with future mortality. Further, quantifying the impact of changes in the level of mortality due to a certain cause of death allows us to develop an appropriate health policy. Particularly, these efforts will provide useful information on actuarial mortality/longevity risk management for life insurance policies, annuity products, and pension plans when investigating various scenarios associated with future mortality and its impact on actuarial valuation.

This case study analyzed cause-of-death mortality data from 2000 to 2016 in Korea and the US. Five major causes of death, which were classified in studies, were observed. The two main causes of death in both countries were cancer and circulatory diseases, which is consistent with previous 

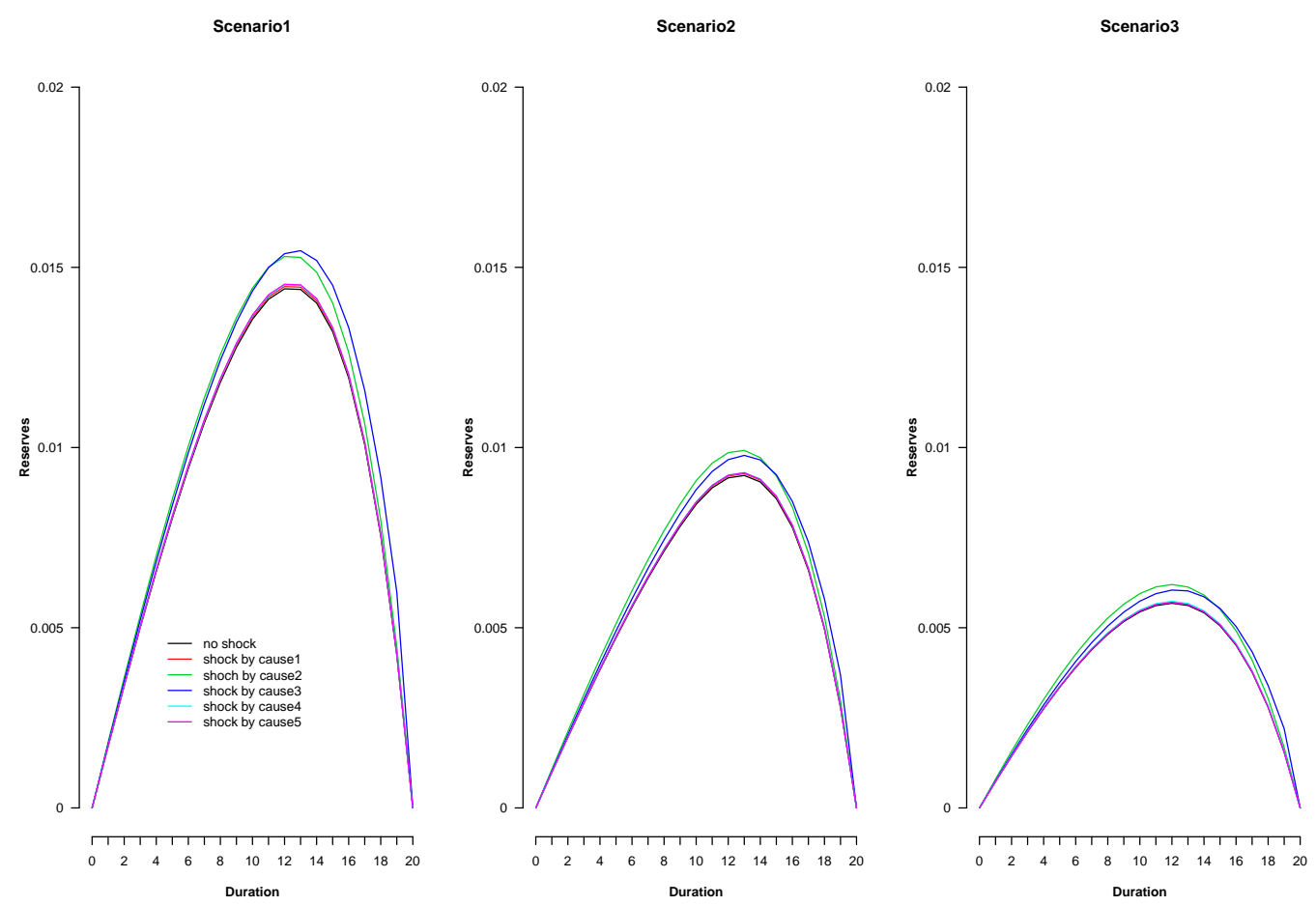

Figure 7: Net premium reserve by duration for 20-year term life insurance (female).

studies. However, it was found that relative mortality levels among five causes and their past trends were fairly different between Korea and US populations for both sexes, which support the results of a former study that cause-of-death mortality should be modeled separately for different countries.

The multinomial logistic regression model has been suggested to model cause-of-death mortality data. The model is simple and easy to implement in practice. The interpretation of the model output is also straight forward. Age is considered a continuous covariate and the number of parameters can be significantly reduced since mortality data is based on each integer age. The age parameter included in the model also allows the evaluation of life expectancy and actuarial values, without any calibration models used in case studies, to derive the 1-year mortality rate from the abridged life table. The multinomial logistic regression models were fitted for Korean data and relevant analysis based on the data were illustrated.

The formula for selected models for males and females were similar and the models fitted the age patterns and the time trends of cause-of-death mortality data. The effects of cause elimination on life expectancy and actuarial values of life insurance and annuity were investigated based on the constructed models. Terms for life expectancy, life insurance policies, and annuity were confined since the period of mortality data is inadequate. Three scenarios were also considered to represent uncertainty on how long the past mortality trend will continue. The analysis of cause-elimination will be useful for actuarial mortality/longevity risk evaluation.

The multinomial logistic regression models must be updated and monitored using updated data for actuarial applications since the patterns of cause-of-mortality were found to change over time and vary by sex and country. Future case studies on the cause-of-death mortality with various population groups will enhance the cause-of-death mortality model, which gives a better understanding of human 
mortality. A more comprehensive understanding of the mechanism of cause-of-death mortality will also be possible if mortality data containing multiple causes of death and correlations is available.

Possible areas for future research includes a study on the cause of death in specific diseases. For example, a study can be performed on cancer mortality by type of cancer to consider the future scenario of cancer mortality, according to the development of cures for specific cancer types. It is also known that there are various risk factors associated with mortality; therefore, the model and risk analysis will improve if several significant mortality risk factors are included in the multinomial logistic regression model as explanatory variables.

\section{References}

Alai DH, Arnold S, and Sherris M (2014). Modelling cause-of-death mortality and the impact of cause-elimination, Annals of Actuarial Science, 9, 167-186.

Arias E, Heron M, and Tejada-Vera B (2013). United States life tables eliminating certain causes of death, 1999-2001, National Vital Statistics Reports, 61, 1-218.

Arnold S and Sherris M (2013). Forecasting mortality trends allowing for cause-of-death mortality dependence, North American Actuarial Journal, 17, 273-282.

Arnold S and Sherris M (2015). Causes-of-death mortality: what do we know on their dependence?, North American Actuarial Journal, 19, 116-128.

Boumezoued A, Labit Hardy H, El Karoui N, and Arnold S (2018). Causes-of-death mortality: What can be learned from population dynamics?, Insurance: Mathematics and Economics, 78, 301315.

De Jong P and Heller GZ (2008). Generalized Linear Models for Insurance Data, Cambridge University Press, New York.

Desesquelles AF, Salvatore MA, Pappagallo M, Frova L, Pace M, Mesle F, and Egidi V (2012). Analysing multiple causes of death: which methods for which data? An application to the cancerrelated mortality in France and Italy, European Journal of Population, 28, 467-498.

Dickson DCM, Hardy MR, and Waters HR (2013). Actuarial Mathematics for Life Contingent Risks (2nd ed), Cambridge University Press, New York.

Dimitrova D, Haberman S, and Kalshev V (2013). Dependent competing risks: cause elimination and its impact on survival, Insurance: Mathematics and Economics, 53, 464-477.

European Insurance and Occupational Pension Authority (2014). Technical Specification for the Preparatory Phase (Part I), 203-204. Available from: https://eiopa.europa.eu

Foreman KJ, Li G, Best N, and Ezzati M (2016). Small area forecasts of cause-specific mortality: application of a Bayesian hierarchical model to US vital registration data, Journal of the Royal Statistical Society (Applied Statistics Series C), 66, 121-139.

Frees E (2010). Regression Modeling with Actuarial and Financial Applications, Cambridge University Press, New York.

Goodman RA, Manton GG, Nolan TF, Bregman DJ, and Hinman AR (1982). Mortality data analysis using a multiple-cause approach, Journal of the American Medical Association, 247, 793-796.

Heron M and Anderson RN (2016). Changes in the leading cause of death: recent patterns in heart disease and cancer mortality, NCHS Data Brief, 254 (August 2016).

Hong L and Yang L (2019). Modeling cause-of-death mortality using hierarchical Archimedean copula, Scandinavian Actuarial Journal, 2019, 247-272.

Israel RA, Rosenberg HM, and Curtin LR (1986). Analytical potential for multiple cause-of-death data, American Journal of Epidemiology, 124, 161-179. 
Manton KG, Tolley HD, and Poss SS (1976). Life table techniques for multiple-cause mortality, Demography, 13, 541-564.

McNown R and Rogers A (1992). Forecasting cause-specific mortality using time series methods, International Journal of Forecasting, 8, 413-432.

Moreno-Betancur M, Sadaoui H, Piffaretti C, and Rey G (2017). Survival analysis with multiple causes of death extending the competing risks model, Epidemiology, 28, 12-19.

Poston DL and Min H (2008). The multinomial regression modeling of the cause-of-death mortality of the oldest old in the U.S., Journal of Modern Applied Statistical Methods, 7, 597-606.

Richards SJ (2009). Selected issues in modelling mortality by cause and in small populations, British Actuarial Journal, 15, 267-283.

Wilmoth JR (1995). Are mortality projections always more pessimistic when disaggregated by cause of death?, Mathematical Population Studies, 5, 293-319.

Yun JW and Son M (2016). Forecasting cause-specific mortality in Korea up to year 2032, Journal of Korean Medical Science, 31, 1181-1189.

Received July 2, 2019; Revised September 4, 2019; Accepted September 27, 2019 